\title{
CONDITIONAL EXPECTATIONS AND AN ISOMORPHIC CHARACTERIZATION OF $L_{1}$-SPACES
}

\section{TZAFRIRI ${ }^{1}$}

Abstract. Conditional expectations can be defined in Banach spaces whose elements can be represented as measurable functions. In the present paper it is shown that such a space (precisely a cyclic space) is isomorphic to an $L_{1}$-space if and only if the conditional expectations act as bounded operators for sufficiently many representations.

Let $(\Omega, \Sigma, \mu)$ be a finite measure space and $\Sigma_{0}$ a subring of $\Sigma$ with maximal element $\Omega_{0}$; then for each $\Sigma$-measurable function $f$ which is bounded on $\Omega$ one can consider the measure $\mu_{0}(\sigma)=\int_{\sigma \cap \Omega_{0}} f(\omega) \mu(d \omega)$; $\sigma \cap \Omega_{0} \in \Sigma_{0}$. Since $\mu_{0}$ is evidently absolutely continuous with respect to the restriction of $\mu$ to the subfield generated by $\Sigma_{0}$ and $\Omega$, due to the Radon-Nikodym theorem, there exists a $\Sigma_{0}$-measurable function denoted $E\left(\Sigma_{0}, \mu\right) f$ for which

$$
\int_{\sigma} f(\omega) \mu(d \omega)=\int_{\sigma} E\left(\Sigma_{0}, \mu\right) f \mu(d \omega) ; \quad \sigma \in \Sigma_{0} .
$$

Obviously the operator $E\left(\Sigma_{0}, \mu\right): f \rightarrow E\left(\Sigma_{0}, f\right) f$ can be extended uniquely to a contractive projection in $L_{p}(\Omega, \Sigma, \mu) ; 1 \leqq p \leqq+\infty$, which is called the conditional expectation relative to $\Sigma_{0}$.

However, if the $L_{p}$-norm is replaced by a general monotonic norm $\rho$ in the sense of the theory of Banach function spaces (see for instance W. A. J. Luxemburg and A. C. Zaanen [9, Note I]), usually, $E\left(\Sigma_{0}, \mu\right)$ does not act as a bounded operator in $L_{\rho}$ - the space of all $\Sigma$-measurable functions for which $\rho(f)<+\infty$, even when we assume that $L_{1}(\Omega, \Sigma, \mu) \supset L_{\rho} \supset L_{\infty}(\Omega, \Sigma, \mu)$. Furthermore, a Banach function space $L_{\rho}$ admits many isometric representations; e.g. to every positive function $\phi \in L_{\rho}$ whose support is $\Omega$ one can define a new norm $\rho_{\phi}(f)$ $=\rho(\phi f)$, obtaining in this way a new Banach function space $L_{\rho_{\phi}}$ which is isometric to $L_{\rho}$ and satisfies $L_{1}(\Omega, \Sigma, \phi \mu) \supset L_{\rho_{\phi}} \supset L_{\infty}(\Omega, \Sigma, \phi \mu)$.

The main result of this paper states that $L_{\rho}$ is isomorphic to an $L_{1}$-space over a finite measure space provided for every subring $\Sigma_{0}$ of

Received by the editors September 24, 1969.

AMS 1969 subject classifications. Primary 4606, 4635, 4725; Secondary 4610, 4720.

Key words and phrases. Conditional expectation, Banach function spaces, cyclic spaces, $L_{1}$-spaces, Boolean algebras of projections, isomorphism between Banach spaces.

Supported in part by National Science Foundation, Grant GP 8217. 
$\Sigma$ and positive function $\phi \in L_{\rho}$ whose support is $\Omega$ the conditional expectation $E\left(\Sigma_{0}, \phi \mu\right)$ is a bounded operator in $L_{\rho_{\phi}}$.

Since a Banach space isomorphic to an $L_{1}$-space is not in general a Banach function space, in order to have a complete characterization we will consider the more natural context (for this purpose) of cyclic spaces introduced by W. G. Bade [3] rather than that of Banach function spaces (for other characterizations using cyclic spaces see [11]).

Conditional expectations in cyclic spaces. We shall start by summarizing some notions and results needed in the sequel. A Boolean algebra of projections $\mathfrak{B}$ in a Banach space $X$ is called $\sigma$-complete (cf. W. G. Bade [2]) provided for every sequence $P_{n} \in \mathfrak{B} ; n=1$, $2, \cdots$, the projections $\bigvee_{n=1}^{\infty} P_{n}$ and $\bigwedge_{n=1}^{\infty} P_{n}$ exist in $\mathscr{B}$ and satisfy

$$
\left(\bigvee_{n=1}^{\infty} P_{n}\right) X=\operatorname{clm}_{n}\left\{P_{n} X\right\} ; \quad\left(\bigwedge_{n=1}^{\infty} P_{n}\right) X=\bigcap_{n=1}^{\infty}\left\{P_{n} X\right\} .
$$

It is well known that $\mathfrak{B}$ can be regarded as a spectral measure $P(\cdot)$ defined on the Borel sets $\Sigma$ of its Stone space $\Omega$ and it follows from W. G. Bade [2, Theorem 2.2] and N. Dunford [4] that there exists a constant $K$ such that for every Borel bounded function $f$, the integral $S(f)=\int_{\Omega} f(\omega) P(d \omega)$ exists in the uniform operator topology and satisfies the inequality: $\|S(f)\| \leqq K \sup _{\omega \in \Omega}|f(\omega)|$. For $f$ unbounded we can consider $S(f)$ as an unbounded operator whose domain is

$$
D(S(f))=\left\{x \mid x \in X, \lim _{m \rightarrow \infty} \int_{\theta_{m}} f(\omega) P(d \omega) x \text { exists }\right\}
$$

and $e_{m}=\{\omega|\omega \in \Omega| f,(\omega) \mid \leqq m\} ; m=1,2, \ldots$ According to W. G. Bade [3], $X$ is a cyclic space relative to a $\sigma$-complete Boolean algebra of projections $\mathfrak{B}$ if there is $x_{0} \in X$ such that $X=\mathfrak{M}\left(x_{0}\right)$ $=\operatorname{clm}\left\{P x_{0} \mid P \in \mathfrak{B}\right\}$. In this case, by W. G. Bade [3, Theorem 4.5], $X=\mathfrak{M}\left(x_{0}\right)=\left\{S(f) x_{0} \mid x_{0} \in D(S(f))\right\}$. Let us also mention that for every cyclic space $X=\mathfrak{M}\left(x_{0}\right)$ there exists a functional $x_{0}^{*} \in X^{*}$, which will be called Bade functional, with the following properties:

(i) $x_{0}^{*} P x_{0} \geqq 0 ; P \in \mathscr{B}$;

(ii) if $x_{0}^{*} P x_{0}=0$ for some $P \in \mathfrak{B}$, then $P=0$ (cf. W. G. Bade [2, Theorem 3.1]).

With this preparation we can state our principal result which is contained in the following theorem.

Theorem 1. A Banach space $X$ is isomorphic to an $L_{1}$-space over a finite measure space if and only if: 
(a) $X$ is a cyclic space $\mathfrak{M}\left(x_{0}\right) ; x_{0} \in X$, relative to some $\sigma$-complete Boolean algebra of projections $\mathfrak{B}$, and

(b) there exists a Bade functional $x_{0}^{*} \in X^{*}$ such that the series

$$
\begin{aligned}
& \sum_{n=1}^{\infty} \frac{x_{0}^{*} P\left(\sigma_{n}\right) S(f) x_{0}}{x_{0}^{*} P\left(\sigma_{n}\right) S(\phi) x_{0}} P\left(\sigma_{n}\right) S(\phi) x_{0} ; \\
& \quad S(f) x_{0}, S(\phi) x_{0} \in \mathfrak{M}\left(x_{0}\right) ; \quad \phi(\omega)>0 ; \quad \omega \in \Omega,
\end{aligned}
$$

converges strongly in $X$ for every sequence of disjoint sets $\sigma_{n} \in \Sigma$; $n=1,2, \cdots$.

Proof. Let $\tau$ be an isomorphism between an $L_{1}$-space $L_{1}(T, J, m)$; $m(T)<+\infty$ and $X ; x_{0}=\tau(1) ; x_{0}^{*}=\left(\tau^{*}\right)^{-1}(1)$ and $\mathcal{F}=\{F(e) \mid e \in T$; $\left.F(e) \tau(f)=\tau\left(\chi_{e} f\right) ; f \in L_{1}(T, J, m)\right\}$. Obviously $X$ is a cyclic space relative to the $\sigma$-complete Boolean algebra of projections $\mathcal{F}$ for which the series in condition (b) of the theorem converges and its sum is bounded in norm by $\|\tau\| \cdot\left\|\tau^{-1}\right\| \cdot\left\|S(f) x_{0}\right\|$.

Conversely, let us set

$$
Q\left(S(f) x_{0}\right)=\sum_{n=1}^{\infty} \frac{x_{0}^{*} P\left(\sigma_{n}\right) S(f) x_{0}}{x_{0}^{*} P\left(\sigma_{n}\right) S(\phi) x_{0}} P\left(\sigma_{n}\right) S(\phi) x_{0} ; \quad S(f) x_{0} \in X .
$$

One can easily see that $Q$ is a linear projection in $X$. In order to show that $Q$ is bounded assume there exist $S\left(f_{n}\right) x_{0} \in X ;\left\|S\left(f_{n}\right) x_{0}\right\|=1$; $n=1,2, \cdots$, such that $\left\|Q\left(S\left(f_{n}\right) x_{0}\right)\right\| \geqq n^{3}$. The inequality

$$
\left\|S\left(\left|f_{n}\right|\right) x_{0}\right\|=\left\|S\left(\frac{\left|f_{n}\right|}{f_{n}}\right) S\left(f_{n}\right) x_{0}\right\| \leqq K\left\|S\left(f_{n}\right) x_{0}\right\|
$$

shows that $S\left(\left|f_{n}\right|\right) x_{0} \in X$ and $\left\|S\left(\left|f_{n}\right|\right) x_{0}\right\| \leqq K ; n=1,2, \cdots$. Set $Q\left(S\left(f_{n}\right) x_{0}\right)=S\left(g_{n}\right) x_{0} ; Q\left(S\left(\left|f_{n}\right|\right) x_{0}\right)=S\left(h_{n}\right) x_{0} ;$ since $\left|g_{n}(\omega)\right| \leqq h_{n}(\omega)$; $\omega \in \Omega$, we obtain

$$
\left\|S\left(h_{n}\right) x_{0}\right\| \geqq \frac{1}{K}\left\|S\left(\left|g_{n}\right|\right) x_{0}\right\| \geqq \frac{1}{K^{2}}\left\|S\left(g_{n}\right) x_{0}\right\| \geqq \frac{n^{3}}{K^{2}} .
$$

Thus for $S(f) x_{0}=\sum_{n=1}^{\infty}\left(S\left(\left|f_{n}\right|\right) x_{0} / n^{2}\right) \in X$ we have

$$
\left\|Q\left(S(f) x_{0}\right)\right\| \geqq \frac{1}{K}\left\|Q\left(\frac{S\left(\left|f_{n}\right|\right) x_{0}}{n^{2}}\right)\right\|=\frac{1}{K n^{2}}\left\|S\left(h_{n}\right) x_{0}\right\| \geqq \frac{n}{K^{3}}
$$

which shows that $Q$ is not defined in $S(f) x_{0} \in X$ i.e. condition (b) of the theorem does not hold. We have to point out that $Q$ depends on the choice of $S(\phi) x_{0} \in X$ and $\sigma_{n} \in \Sigma ; n=1,2, \cdots$. 
Now, let $\left\{\delta_{n}^{\prime}\right\}$ and $\left\{\delta_{n}^{\prime \prime}\right\}$ be two sequences of mutually disjoint sets; $\delta_{n}^{\prime}, \delta_{n}^{\prime \prime} \in \Sigma ; n=1,2, \cdots,\left(\cup_{n=1}^{\infty} \delta_{n}^{\prime}\right) \cap\left(\cup_{n=1}^{\infty} \delta_{n}^{\prime \prime}\right)=\varnothing$ for which $P\left(\delta_{n}^{\prime}\right)$ as well as $P\left(\delta_{n}^{\prime \prime}\right)$ are nonzero projections. Set $\mu(\sigma)=x_{0}^{*} P(\sigma) x_{0}$; $\sigma \in \Sigma ; a_{n}=\min \left\{\mu\left(\delta_{n}^{\prime}\right), \mu\left(\delta_{n}^{\prime \prime}\right)\right\}$ and

$$
\begin{aligned}
S(\phi) x_{0}=\sum_{n=1}^{\infty} & \frac{a_{n}}{2^{n}} \frac{P\left(\delta_{n}^{\prime}\right) x_{0}}{\mu\left(\delta_{n}^{\prime}\right)}+\sum_{n=1}^{\infty} \frac{a_{n}}{2^{n}} \frac{P\left(\delta_{n}^{\prime \prime}\right) x_{0}}{\mu\left(\delta_{n}^{\prime \prime}\right)} \\
& +P\left(\Omega-\left(\bigcup_{n=1}^{\infty} \delta_{n}^{\prime} \cup \bigcup_{n=1}^{\infty} \delta_{n}^{\prime \prime}\right)\right) x_{0} .
\end{aligned}
$$

Since $\phi(\omega)>0 ; \omega \in \Omega$, we can consider the projection $Q$ corresponding to $S(\phi) x_{0}$ and the partition $\sigma_{n}=\delta_{n}^{\prime} \cup \delta_{n}^{\prime \prime} ; n=1,2, \cdots$. Then

$$
Q\left(P\left(\delta_{n}^{\prime}\right) x_{0}\right)=\frac{2^{n-1}}{a_{n}} \mu\left(\delta_{n}^{\prime}\right)\left[\frac{a_{n}}{2^{n}} \frac{P\left(\delta_{n}^{\prime}\right) x_{0}}{\mu\left(\delta_{n}^{\prime}\right)}+\frac{a_{n}}{2^{n}} \frac{P\left(\delta_{n}^{\prime \prime}\right) x_{0}}{\mu\left(\delta_{n}^{\prime \prime}\right)}\right]
$$

and further

$$
Q\left(\frac{P\left(\delta_{n}^{\prime}\right) x_{0}}{\mu\left(\delta_{n}^{\prime}\right)}\right)=\left(\frac{P\left(\delta_{n}^{\prime}\right) x_{0}}{\mu\left(\delta_{n}^{\prime}\right)}+\frac{P\left(\delta_{n}^{\prime \prime}\right) x_{0}}{\mu\left(\delta_{n}^{\prime \prime}\right)}\right) / 2
$$

and similarly:

$$
Q\left(\frac{P\left(\delta_{n}^{\prime \prime}\right) x_{0}}{\mu\left(\delta_{n}^{\prime \prime}\right)}\right)=\left(\frac{P\left(\delta_{n}^{\prime}\right) x_{0}}{\mu\left(\delta_{n}^{\prime}\right)}+\frac{P\left(\delta_{n}^{\prime \prime}\right) x_{0}}{\mu\left(\delta_{n}^{\prime \prime}\right)}\right) / 2 .
$$

This implies that a series $\sum_{n=1}^{\infty} c_{n} P\left(\delta_{n}^{\prime}\right) x_{0} / \mu\left(\delta_{n}^{\prime}\right)$ converges if and only if the series $\sum_{n=1}^{\infty} c_{n} P\left(\delta_{n}^{\prime \prime}\right) x_{0} / \mu\left(\delta_{n}^{\prime \prime}\right)$ does, i.e., the basis $\left\{P\left(\delta_{n}^{\prime}\right) x_{0} / \mu\left(\delta_{n}^{\prime}\right)\right\}$ is equivalent to the basis $\left\{P\left(\delta_{n}^{\prime \prime}\right) x_{0} / \mu\left(\delta_{n}^{\prime \prime}\right)\right\}$. If $\pi$ is a permutation of the natural numbers, the bases $\left\{P\left(\delta_{n}^{\prime}\right) x_{0} / \mu\left(\delta_{n}^{\prime}\right)\right\}$ and $\left\{P\left(\delta_{\pi(n)}^{\prime}\right) x_{0} / \mu\left(\delta_{\pi(n)}^{\prime}\right)\right\}$ will be equivalent since both are equivalent to $\left\{P\left(\delta_{n}^{\prime \prime}\right) x_{0} / \mu\left(\delta_{n}^{\prime \prime}\right)\right\}$. In the terminology of I. Singer [10] (see also M. J. Kadec and A. Pełczynski [7]) this means that both bases $\left\{P\left(\delta_{n}^{\prime}\right) x_{0} / \mu\left(\delta_{n}^{\prime}\right)\right\}$ and $\left\{P\left(\delta_{n}^{\prime \prime}\right) x_{0} / \mu\left(\delta_{n}^{\prime \prime}\right)\right\}$ are symmetric and thus by [7, Theorem 5] there exists $M^{\prime}$ and $M^{\prime \prime}$ such that

$$
\frac{1}{\left\|x_{0}^{*}\right\|} \leqq \frac{\left\|P\left(\delta_{n}^{\prime}\right) x_{0}\right\|}{\mu\left(\delta_{n}^{\prime}\right)} \leqq M^{\prime} ; \quad \frac{1}{\left\|x_{0}^{*}\right\|} \leqq \frac{\left\|P\left(\delta_{n}^{\prime \prime}\right) x_{0}\right\|}{\mu\left(\delta_{n}^{\prime \prime}\right)} \leqq M^{\prime \prime} ; \quad n=1,2, \cdots
$$

Consequently, for any partition $\left\{\delta_{n}\right\}$ there exists $M$ such that $\left\|P\left(\delta_{n}\right) x_{0}\right\| / \mu\left(\delta_{n}\right) \leqq M ; n=1,2, \cdots$, (since we can take $\delta_{n}^{\prime}=\delta_{2 n-1}$ and $\left.\delta_{n}^{\prime \prime}=\delta_{2 n} ; n=1,2, \cdots\right)$.

The next step will be to show that for any partition $\left\{\delta_{n}\right\}$ the basis $\left\{P\left(\delta_{n}\right) x_{0} / \mu\left(\delta_{n}\right)\right\}$ is equivalent to the natural basis of $l_{1}$. Indeed, if a series $\sum_{n=1}^{\infty} c_{n} P\left(\delta_{n}\right) x_{0} / \mu\left(\delta_{n}\right)$ is convergent, then it is easy to see that 
the series $\sum_{n=1}^{\infty}\left|c_{n}\right| P\left(\delta_{n}\right) x_{0} / \mu\left(\delta_{n}\right)$ is convergent too. By repeating the previous part of the proof for

$$
S(\psi) x_{0}=\sum_{n=1}^{\infty}\left|c_{n}\right| \frac{P\left(\delta_{n}\right) x_{0}}{\mu\left(\delta_{n}\right)}+P\left(\Omega-\bigcup_{n=1}^{\infty} \delta_{n}\right) x_{0}
$$

instead of $x_{0}$ (which is possible since $\psi(\omega)>0 ; \omega \in \Omega$ ) we get $\sum_{n=1}^{\infty}\left\|P\left(\delta_{n}\right) S(\psi) x_{0}\right\|<+\infty$ which implies the convergence of $\sum_{n=1}^{\infty}\left|c_{n}\right|$. Since the converse is obvious the assertion is completely proved.

The crucial point in the proof is to show that

$$
\sup _{0 \neq \delta \in \Sigma} \frac{\left\|P(\delta) x_{0}\right\|}{\mu(\delta)}<+\infty \text {. }
$$

Suppose there exists a sequence $\eta_{n} \in \Sigma$, for which $\left\|P\left(\eta_{n}\right) x_{0}\right\| / \mu\left(\eta_{n}\right) \geqq n$; $n=1,2, \cdots$, and $\mu\left(\eta_{n}\right) \neq 0$. We shall construct by induction another sequence $\left\{\sigma_{n}\right\}$ with the properties

(i) $\sigma_{n} \cap \sigma_{i}$ is equal to $\sigma_{n}$ or $\varnothing$;

(ii) $\left\|P\left(\sigma_{n}\right) x_{0}\right\| / \mu\left(\sigma_{n}\right) \geqq n ; 1 \leqq i \leqq n-1$.

Indeed, set $\sigma_{1}=\eta_{1}$ and assume that $\sigma_{1}, \cdots, \sigma_{n}$ are already constructed and satisfying the conditions (i) and (ii). The following equality

$$
\eta_{n+1}=\left(\eta_{n+1}-\bigcup_{k=1}^{n} \sigma_{k}\right) \cup\left(\eta_{n+1} \cap \sigma_{n}\right) \cup \bigcup_{k=1}^{n-1}\left(\eta_{n+1} \cap\left(\sigma_{k}-\bigcup_{j=k+1}^{n} \sigma_{j}\right)\right)
$$

splits $\eta_{n+1}$ in to $n+1$ disjoint sets; hence

$$
\begin{aligned}
\left\|P\left(\eta_{n+1}-\bigcup_{n=1}^{n} \sigma_{k}\right) x_{0}\right\| & +\left\|P\left(\eta_{n+1} \cap \sigma_{n}\right) x_{0}\right\| \\
& \quad+\sum_{k=1}^{n-1}\left\|P\left(\eta_{n+1} \cap\left(\sigma_{k}-\bigcup_{j=k+1}^{n} \sigma_{j}\right)\right) x_{0}\right\| \\
\geqq & \left\|P\left(\eta_{n+1}\right) x_{0}\right\| \geqq(n+1) \mu\left(\eta_{n+1}\right) \\
= & (n+1)\left[\mu\left(\eta_{n+1}-\bigcup_{k=1}^{n} \sigma_{k}\right)+\mu\left(\eta_{n+1} \cap \sigma_{n}\right)\right. \\
& \left.\quad+\sum_{k=1}^{n-1} \mu\left(\eta_{n+1} \cap\left(\sigma_{k}-\bigcup_{j=k+1}^{n} \sigma_{j}\right)\right)\right] .
\end{aligned}
$$

Thus, at least for one of these disjoint sets, which will be denoted $\eta_{n+1}$ we have $\left\|P\left(\eta_{n+1}\right) x_{0}\right\| \geqq(n+1) \mu\left(\sigma_{n+1}\right)$ and in this way all conditions imposed on $\left\{\sigma_{n}\right\}$ will hold. 
Condition (i) satisfied by the sequence $\left\{\sigma_{n}\right\}$ shows that it contains either a subsequence of disjoint sets or a nested subsequence $\left\{\sigma_{n_{j}}\right\}$. The first possibility leads immediately to a contradiction of condition (ii) while in the second case we will have the basis

$$
\left\{P\left(\sigma_{n_{j}}-\sigma_{n_{j+1}}\right) x_{0} / \mu\left(\sigma_{n_{j}}-\sigma_{n_{j+1}}\right)\right\}
$$

which is equivalent to the natural basis of $l_{1}$. In view of the closed graph theorem this implies the existence of a constant $A$ such that:

$$
\left\|\sum_{j=1}^{\infty} \alpha_{j} \frac{P\left(\sigma_{n_{j}}-\sigma_{n_{j+1}}\right) x_{0}}{\mu\left(\sigma_{n_{j}}-\sigma_{n_{j+1}}\right)}\right\| \leqq A \sum_{j=1}^{\infty}\left|\alpha_{j}\right|
$$

for any sequence $\left(\alpha_{j}\right) \in l_{1}$. Taking $\alpha_{j}=\mu\left(\sigma_{n_{j}}-\sigma_{n_{j+1}}\right) / \mu\left(\sigma_{n_{k}}-\sigma_{n_{m+1}}\right)$; $k \leqq j \leqq m$, we obtain

$$
\left\|\sum_{j=k}^{m} \frac{P\left(\sigma_{n_{j}}-\sigma_{n_{j+1}}\right) x_{0}}{\mu\left(\sigma_{n_{k}}-\sigma_{n_{m+1}}\right)}\right\| \leqq A
$$

i.e.

$$
\left\|\frac{P\left(\sigma_{n_{k}}\right) x_{0}-P\left(\sigma_{n_{m+1}}\right) x_{0}}{\mu\left(\sigma_{n_{k}}\right)-\mu\left(\sigma_{n_{m+1}}\right)}\right\| \leqq A .
$$

But $\mu\left(\sigma_{n_{m+1}}\right) \leqq K\left\|x_{0}\right\| / n_{m+1}$ i.e. $\lim _{m \rightarrow \infty} \mu\left(\sigma_{n_{m+1}}\right)=0$ and therefore $\lim _{m \rightarrow \infty}\left\|P\left(\sigma_{n_{m+1}}\right) x_{0}\right\|=0$. Thus $\left\|P\left(\sigma_{n_{k}}\right)\right\| / \mu\left(\sigma_{n_{k}}\right) \leqq A ; \quad k=1,2, \cdots$, which contradicts again condition (ii).

In conclusion we have proved the existence of a constant $L$ such that

$$
\left\|P(\delta) x_{0}\right\| / \mu(\delta) \leqq L ; \quad \delta \in \Sigma ; \mu(\delta) \neq 0 .
$$

Finally, let $f$ be a simple function; one can easily see that

$$
\begin{aligned}
\frac{1}{K\left\|x_{0}^{*}\right\|} \int_{\Omega}|f(\omega)| \mu(d \omega) & \leqq \frac{1}{K}\left\|S(|f|) x_{0}\right\| \leqq\left\|S(f) x_{0}\right\| \\
& \leqq L \int_{\Omega}|f(\omega)| \mu(d \omega)
\end{aligned}
$$

which shows that $X=\mathfrak{M}\left(x_{0}\right)$ is isomorphic to $L_{1}(\Omega, \Sigma, \mu)$.

Let $X=\mathfrak{M}\left(x_{0}\right)$ be a cyclic space relative to a $\sigma$-complete Boolean algebra of projections $\mathfrak{B}$ (regarded as a spectral measure $P(\cdot)$ on $(\Omega$, $\Sigma)$ and $x_{0}^{*}$ a Bade functional. It is quite clear that for any $x_{\phi}=S(\phi) x_{0}$ $\in X ; \phi(\omega)>0 ; \omega \in \Omega$, we have $\mathfrak{M}\left(x_{0}\right)=\mathfrak{M}\left(x_{\phi}\right)$. Considering the positive measure $\nu_{\phi}(\sigma)=x_{0}^{*} P(\sigma) x_{\phi}$ we can define the conditional expectation $E\left(\Sigma_{0}, \nu_{\phi}\right)$ relative to a subring $\Sigma_{0}$ of $\Sigma$ as the operator in $\mathfrak{M}\left(x_{\phi}\right)$ 
$=X$ which assigns to $S(f) x_{\phi} \in \mathfrak{M}\left(x_{\phi}\right)$ the vector $E\left(\Sigma_{0}, \nu_{\phi}\right) S(f) x_{\phi}$ $=S(h) x_{\phi}$ where $h$ would be the Radon-Nikodym derivative of the measure $x_{0}^{*} P(\sigma) S(f) x_{\phi} ; \sigma \in \Sigma_{0}$, with respect to the restriction of $\nu_{\phi}$ to $\Sigma_{0}\left(S(f) x_{\phi}\right.$ belongs to the domain of $E\left(\Sigma_{0}, \nu_{\phi}\right)$ if and only if $\left.x_{\phi} \in D(S(h))\right)$.

Now the previous theorem can be restated as follows:

TheOREM 2. A Banach space $X$ is isomorphic to an $L_{1}$-space over a finite measure space if and only if:

(a) $X$ is a cyclic space $\mathfrak{M}\left(x_{0}\right) ; x_{0} \in X$, relative to some $\sigma$-complete Boolean algebra of projections $\mathfrak{B}$, and

(b) there exists a Bade functional $x_{0}^{*} \in X^{*}$ such that for every subring $\Sigma_{0}$ of $\Sigma$ and $x_{\phi}=S(\phi) x_{0} \in X ; \phi(\omega)>0 ; \omega \in \Omega$, the conditional expectation $E\left(\Sigma_{0}, \nu_{\phi}\right)$ is a linear bounded projection in $X=\mathfrak{M}\left(x_{\phi}\right)$.

Proof. It suffices to observe that the series involved in the statement of Theorem 1 converges and its sum is $E\left(\Sigma_{0}, \nu_{\phi}\right) S\left(f \phi^{-1}\right) x_{\phi}$ where $\Sigma_{0}$ is the subring generated by the sets $\sigma_{n} ; n=1,2, \cdots$ Q.E.D.

REMARKs. 1. Using Banach function spaces instead of cyclic spaces might simplify the statement of Theorem 2 but only a sufficient condition for such spaces to be isomorphic to an $L_{1}$-space can be obtained. The precise assertion appears in the introduction.

2. In defining the measures $\nu_{\phi}$ we use the same Bade functional $x_{0}^{*}$; if instead we set $\lambda_{\phi}(\cdot)=x_{\phi}^{*} P(\cdot) x_{\phi}$, where $x_{\phi}^{*}$ depends on $x_{\phi}, L_{\rho}$ will be isometric to an $L_{p}$-space; $1 \leqq p<+\infty$, provided all the conditional expectations $E\left(\Sigma_{0}, \lambda_{\phi}\right)$ will be contractive projections in $L_{\rho}$ (cf. T. Ando [1]).

3. The problems discussed in this paper are related to the so called "leveling property" of a norm $\rho$ in a Banach function space (cf. H. W. Ellis and I. Halperin [5]) and to the property $(\mathrm{J})$ introduced by N. E. Gretsky [6]. It follows from Theorem 1 that unless a weakly sequentially complete Banach function space is isomorphic to an $L_{1}$ space, there exists always an isometric representation $L_{\rho_{\phi}}$ of $L_{\rho}$ in which $\sup _{\phi} \rho_{\phi}(f)=+\infty$ (in the notation of [6]), and consequently $\rho_{\phi}$ does not admit an equivalent rearrangement-invariant norm with respect to the measure $\nu_{\phi}$ (cf. W. A. J. Luxemburg [8, Theorem 14.4]).

\section{REFERENCES}

1. T. Ando, Banachverbände und positive Projektionen, Math. Z. 109 (1969), 121130.

2. W. G. Bade, On Boolean algebras of projections and algebras of operators, Trans. Amer. Math. Soc. 80 (1955), 345-360. MR 17, 513.

3. - A multiplicity theory for Boolean algebras of projections in Banach spaces, Trans. Amer. Math. Soc. 92 (1959), 508-530. MR 21 \#7443. 
4. N. Dunford, Spectral operators, Pacific J. Math. 4 (1954), 321-354. MR 16, 142.

5. H. W. Ellis and I. Halperin, Function spaces determined by a levelling length function, Canad. J. Math. 5 (1953), 576-592. MR 15, 439.

6. N. E. Gretsky, Representation theorems on Banach function spaces, Bull. Amer. Math. Soc. 74 (1968), 705-709. MR 37 \#2009.

7. M. I. Kadec and A. Pelczynski, Bases, lacunary sequences and complemented subspaces in the spaces $L_{p}$, Studia Math. 21 (1961/62), 161-176. MR 27 \#2851.

8. W. A. J. Luxemburg, Rearrangement-invariant Banach function spaces, Proc. Sympos. Anal. (Queen's Univ., 1967), Queen's Papers in Pure and Appl. Math., no. 10, 1967, pp. 88-144.

9. W. A. J. Luxemburg and A. C. Zaanen, Notes on Banach function spaces. I, Nederl. Akad. Wetensch. Proc. Ser. A $66=$ Indag. Math. 25 (1963), 135-147. MR 26 \#6723a.

10. I. Singer, Some characterizations of symmetric bases in Banach spaces, Bull. Acad. Polon. Sci. Sér. Sci. Math. Astronom. Phys. 10 (1962), 185-192. MR 26 \#5393.

11. L. Tzafriri, $A n$ isomorphic characterization of $L_{p}$ and $c_{0}$-spaces, Studia Math. 32 (1969), 287-296.

NorthWESTERN University, Evanston, IllinoIs 60201

University of Washington, Seattle, Washington 98105 